\title{
The effect of a mesenchymal stem cell conditioned medium fraction on morphological characteristics of hepatocytes in acetaminophen-induced acute liver failure: a preliminary study
}

This article was published in the following Dove Press journal:

Hepatic Medicine: Evidence and Research

\begin{abstract}
Andrey Temnov ${ }^{1,2}$
Konstantin Rogov ${ }^{3,4}$

Vitaliy Zhalimov'

Popov Igor ${ }^{2}$

Stanislav Pekov ${ }^{2}$

Augustinus Bader ${ }^{5}$

Alla Sklifas'

Shibashish Giri ${ }^{5,6}$

'Department of Biology, Institute of Cell Biophysics of the Russian Academy of Sciences, Pushchino, Russia; ${ }^{2}$ Department of Biotechnology, Moscow Institute of Physics and Technology, Dolgoprudny, Russia; ${ }^{3}$ Department of Clinical Morphology, Research Institute of Human Morphology, Moscow, Russia;

${ }^{4}$ Department of Pathomorphology, Peoples' Friendship University of Russia, Moscow, Russia; ${ }^{5}$ Applied Stem Cell Biology and Cell Technology, Biomedical and Biotechnological Center, Leipzig University, Leipzig, Germany;

${ }^{6}$ Department of Plastic and Hand Surgery, University Hospital Rechts der Isar, Munich Technical University, Munich, Germany
\end{abstract}

Correspondence: Shibashish Giri Applied Stem Cell Biology and Cell Technology, Biomedical and Biotechnological Center (BBZ), Medical Faculty, University of Leipzig, Deutscher Platz 5, Leipzig D-04103, Germany Tel +49 34I 973 I353; +49 I74 7834363 Fax $+49341973 \quad 1329$

Email Shibashish.giri@bbz.uni-leipzig.de
Background: In our studies, it was shown that the effectiveness of the conditioned medium obtained by cultivating mesenchymal stem cells depends on the microenvironment conditions used to cultivate the cells. It was demonstrated that the conditioned medium obtained by culturing cells with low oxygen content $(10 \%)$ has a much more pronounced protective effect. Methods: Protein compositions obtained from MSCs cultured under hypoxic $\left(10 \% \mathrm{O}_{2}\right.$ hcMSC) and normal $\left(21 \% \mathrm{O}_{2} \mathrm{nc}-\mathrm{MSC}\right)$ conditions were used to treat acute liver failure (ALF) induced in mice by acetaminophen injection. Thus, we obtained fractions normalized by volume, which predominantly contained proteins with masses $>50,50-30,30-10$, and 10-3 kDa.

Results: The data from biochemical studies have shown that only fractions from 10 to 30 $\mathrm{kDa}$ (hcMSC and ncMSC) significantly reduced the level of liver enzymes in the beginning of the acute period after acetaminophen administration. Mass spectrometry analysis of the proteins contained in the isolated fractions showed a sharp increase in the protein levels in the 10-30 kDa hcMSC fraction as compared with that in 10-30 kDa ncMSCs. The composition obtained from MSCs cultured at lower $\mathrm{O}_{2}$ level (fraction 10-30 kDa hcMSC) was shown to be more potent than the composition prepared from normoxic cells.

Conclusion: The results have shown that a composition obtained by culturing the cells under a reduced content of $\mathrm{O}_{2}(10 \%)$, significantly improves the biochemical parameters, and histological arrester reduces the degree of inflammation and stimulates regenerative processes in liver, compared to both the control group and group treated with the composition that was obtained by culturing the cells under normal oxygen content.

Keywords: acute hepatic failure, stem cells, condition media, galectin-1, mass spectrometry

\section{Introduction}

Acute liver failure (ALF) is a condition where hepatocyte death occurs within days or weeks without cirrhosis signs. The death can be caused by numerous agents, including viruses, toxins, and drugs. In the United States, approximately 2,000 people are diagnosed with ALF every year, and almost $60 \%$ of the cases are caused by acetaminophen (APAP) or idiosyncratic drug reactions caused by drugs. ${ }^{1}$ In general, APAP-induced injury significantly exceeds idiosyncratic drug-induced liver injury, with their approximate ratio being 4:1.

Most APAP-associated ALF cases have signs of suicide; however, as early as the 1980s, there were reported cases of the ALF development associated with therapeutic drug administration or alcohol-tylenol syndrome. ${ }^{2,3}$ 
$\mathrm{N}$-acetylcysteine (NAC) is the best treatment for paracetamol poisoning. Since the 1970s, NAC therapy has been the best therapeutic option for treating patients with APAP-induced ALF. However, various adverse reactions to NAC, with their severity ranging from minor to fatal, have been reported in the clinic. ${ }^{4}$ In addition, the therapeutic window for the introduction of NAC is rather narrow, while some patients already have signs of ALF. Finally, NAC binds toxic NAPQI, but its effectiveness is drastically reduced in the late stages of the pathological process. It is also known that NAC is much less effective in APAP-treated mice. ${ }^{5}$ Consequently, the development of new drugs designed to suppress the progression of APAPinduced hepatitis is of great importance and would be highly desirable.

One of the effective ways of treating ALF developed due to APAP administration is transplantation of mesenchymal stem cells. ${ }^{6,7}$ A number of studies have demonstrated that transplantation causes an anti-inflammatory, antiapoptotic, and proliferative effect on mice. ${ }^{8,9}$

However, some authors believe that the clinical effect caused by the introduction of stem cells is primarily associated with paracrine factors released by cells into culture medium. In this case, introduction of conditioned medium derived from stem cells exerts an effect that is only slightly inferior to that of MSC introduction. ${ }^{10}$

In our previous studies, we demonstrated that the effectiveness of conditioned medium obtained during cultivation of mesenchymal stem cells depended on the microenvironment conditions used for cultivation of the cells. ${ }^{11}$ For example, conditioned medium obtained during cultivation of cells under low oxygen content (10\%) condition was demonstrated to have a significantly more pronounced protective effect. ${ }^{9}$

The purpose of this study was to isolate a molecular fraction of the conditioned medium, which possessed the maximum protective effect in APAP-induced liver failure, and to characterize changes, using mass spectrometry, in the qualitative and quantitative composition of proteins, depending on cultivation conditions.

\section{Materials and methods}

\section{Preparation of bone marrow stem cells}

To isolate MSCs, the bone marrow was harvested from the femur of CD1 mice under general anesthesia. The mononuclear fraction of bone marrow cells was isolated on a density gradient using a standard Lympholyte-H solution (Cedarlane,
Canada). After preparation, the suspension of mononuclear cells was seeded on Petri dishes and cultured in DMEM medium with addition of $10 \%$ fetal calf serum (Gibco).

The produced cells were subjected to osteogenic, chondrogenic, and adipogenic differentiation according to the standard technique to confirm the fact that they possessed the MSC properties. ${ }^{12}$

Osteogenic differentiation (with addition of $10 \%$ fetal calf serum, $100 \mu \mathrm{M}$ dexamethasone, $0.1 \mathrm{mM}$ ascorbic acid, and 10 $\mathrm{nM} \beta$-glycerophosphate) was confirmed by the presence of alkaline phosphatase in cell culture using standard reagents (Sigma-Aldrich, USA). Chondrogenic differentiation (with addition of $10 \%$ fetal calf serum, $100 \mu \mathrm{M}$ dexamethasone, and $0.1 \mu \mathrm{g} / \mathrm{mL}$ TGF- $\beta$ ) was confirmed by alcian blue staining. Adipogenic differentiation (with addition of $10 \%$ fetal calf serum, $10 \mu \mathrm{M}$ dexamethasone, $10 \mu \mathrm{g} / \mathrm{mL}$ insulin, and 100 $\mu \mathrm{g} / \mathrm{mL}$ IBMX) was confirmed by Oil Red-O staining.

\section{MSC cultivation}

A cell monolayer was cultivated under normoxia $\left(21 \% \mathrm{O}_{2}\right.$ and $5 \% \mathrm{CO}_{2}$ ncMSC) and hypoxia $\left(10 \% \mathrm{O}_{2}\right.$ and $5 \% \mathrm{CO}_{2}$ hcMSC) conditions. We could not see any differences between the cells; the stabs were cultivated under conditions of normoxia (CD44 98.2\%, CD90 97.6\%, CD31 $1.0 \%$, and CD45 0.6\%) and hypoxia (CD44 97.0\%, CD90 97.3\%, CD31 1.1\%, and CD45 0.8\%).

\section{CMSC preparation}

After preparing the cell monolayer, culture medium was completely refreshed, and, after 3 days, conditioned culture medium was combined with the MSC lysate (1:1). The conditioned medium derived from MSCs cultured under normoxia conditions was designated as ncMSC, and the conditioned medium obtained from MSCs cultured under hypoxia conditions was designated as hcMSC.

\section{Fraction preparation}

Ultrafiltration was used to separate conditioned medium. 10 $\mathrm{mL}$ of studied conditioned medium was placed in an Amicon Ultra-15 50,000 NMWL filter device (Millipore, Ireland) and centrifuged using a swinging-bucket rotor at $+4^{\circ} \mathrm{C}$ for 25 mins. The upper fraction was washed three times with 10 $\mathrm{mL}$ of $1 \mathrm{X}$ PBS (Amresco, USA), transferred to a new tube, and adjusted to a volume of $10 \mathrm{~mL}$ with $1 \mathrm{X}$ PBS. The filtrate obtained during the first filtration was transferred to a 30,000 NMWL concentrator. The filtration procedure was carried out as described above, except that the upper fraction was diluted to the filtrate volume initially introduced into the 
concentrator. Thus, we obtained fractions normalized by volume, which contained predominantly proteins with masses $>50,50-30,30-10$, and 10-3 kDa.

\section{Mass spectrometry}

The protein solution $\mathrm{pH}$ was adjusted to 8.0 by adding $1 \mathrm{M}$ Tris-Cl, followed by addition of $5 \%$ acetonitrile to facilitate further hydrolysis. $50 \mu \mathrm{L}$ of the protein solution was mixed with $5 \mu \mathrm{L}$ of $50 \mathrm{mM}$ DTT and incubated at $65^{\circ} \mathrm{C}$ for $5 \mathrm{mins}$, followed by addition of $5 \mu \mathrm{L}$ of $100 \mathrm{mM}$ IAA and incubation at $30^{\circ} \mathrm{C}$ in a dark place for 30 mins. Then, $5 \mu \mathrm{L}$ of $1 \mu \mathrm{g} / \mu \mathrm{L}$ sequencing grade trypsin (Promega) was added and incubated at $37^{\circ} \mathrm{C}$ overnight. The reaction was stopped by adding $5 \mu \mathrm{L}$ of $0.5 \%$ acetic acid. The reaction mixture was centrifuged at $12,500 \mathrm{~g}$ for $10 \mathrm{mins}$, and the supernatant was transferred to a new tube and stored at $-20^{\circ} \mathrm{C}$ until further analysis.

Peptide mixtures were analyzed on a nano-HPLC Agilent 1100 system (Agilent Technologies, Santa Clara, CA, USA) combined with a 7T LTQ-FT Ultra mass spectrometer (Thermo Electron, Bremen, Germany) equipped with a nanospray ion source (positive mode). Peptides were separated on a homemade capillary column (Fused-silica PicoTip $^{\circledR}$; emitter id, $75 \mu \mathrm{m}$; length, $12 \mathrm{~cm}$; nominal tip id, $15 \mu \mathrm{m}$ ) packed with Reprosil-Pur Basic C18, $3 \mu \mathrm{m}, 100 \mathrm{~A}$ (Dr Maisch HPLC GmbH, Ammerbuch-Entringen, Germany). The separation was carried out using a 120-min gradient $\left(\mathrm{H}_{2} \mathrm{O} / \mathrm{ACN}\right.$ containing $0.1 \%$ formic acid) from $3 \%$ to $90 \%$ of $\mathrm{ACN}$ at a flow rate of $300 \mathrm{~nL} / \mathrm{min}$. MS and MS/MS data were acquired in a data-dependent mode using the Xcalibur (Thermo Finnigan, San Jose, USA) software.

MS data were analyzed using the MaxQuant program against the SwissProt database with an initial precursor mass error of $10 \mathrm{ppm}$. The minimum peptide length for identification was set to seven amino acids; the "match between runs" option was activated, and the cutoff false discovery rate was set to 0.01 . Proteins with more than two peptides and with at least one unique peptide were considered as identified. For protein quantification, label-free analysis was used.

\section{Simulation of ALF}

Male mice of the BaLb line weighing 22-24 g were divided into two groups (the control and experimental ones). APAP was administered intraperitoneally at a dose of $270 \mathrm{mg} / \mathrm{kg}$ to animals of all groups. After 15 mins, animals in the control group (No. 1, n=15) were intraperitoneally injected with $1 \mathrm{~mL}$ of intact culture medium (DMEM supplemented with 10\% fetal calf serum without culturing any cells), whereas all animals in the experimental group (group No. $2=15$ and No. $3 \mathrm{n}=15$ ) received $1 \mathrm{~mL}$ of $\mathrm{ncMSC}$ or hcMSC compositions ( $0.5 \mathrm{~mL}$ condition media $+0.5 \mathrm{~mL}$ lysate $\mathrm{MSC}$, protein concentration, $10 \mathrm{mg} / \mathrm{mL}$ ).

\section{Histology}

Material for histological examination was collected $4 \mathrm{hrs}$ after APAP administration. For morphological assessment of changes, the liver of mice was fixed in $10 \%$ neutral buffered formalin, and 4-5 $\mu \mathrm{m}$ thick serial paraffin sections were prepared.

Dystrophic and necrotic changes in the liver were scored based on analysis of 10 fields of view using a semi-quantitative blind method. ${ }^{13}$ The following scale was used: 0 - no changes; 0.5 - dystrophic changes in $<1 / 3$ of hepatocytes; 1 - dystrophic changes in more than $1 / 3$ of hepatocytes; 2 - dystrophic changes in more than $2 /$ 3 of hepatocytes; 3 - dystrophic changes in 100\% of hepatocytes; 4 - the sample contains a combination of dystrophy with single focal necroses; and 5 - extensive focal necrosis. For scored assessment, six animals in each group were examined.

\section{Biochemical study}

The levels of liver enzymes GPT and GOT were measured in intact animals before administration of APAP. The effect of the abovementioned composition was evaluated based on changes in the serum levels of ALS and GOT in animals $4 \mathrm{hrs}$ after administration of APAP. The serum levels of GOT and GPT were determined using the kinetic method on an Olympus AU2700 biochemical analyzer (Beckman Coulter, USA).

\section{Statistical analysis}

Statistical analysis was performed using the Statistica 6.0 software. The Mann-Whitney U-test was used for nonparametric data. Differences were considered statistically significant at $p<0.05$.

\section{Ethical considerations}

Ethical clearance for the study was obtained from the Institute of Cell Biophysics of the Russian Academy of Sciences prior to study initiation. The study was conducted in compliance with the principles of the Declaration of Helsinki. In addition to the approval from the Institute of Cell Biophysics of the Russian Academy of Sciences, the maintenance and ethical treatment of laboratory animals was done in accordance with 
Guidelines of the European Convention for the Protection of Vertebrate animals used for experiments and Guidelines for working with laboratory animals in ICB RAS, Order N39 from December 30, 2018.

\section{Results}

\section{Biochemical study}

The biochemical study data demonstrated that only 10-30 $\mathrm{kDa}$ fractions (hcMSC and ncMSC) significantly reduced the level of hepatic enzymes in the acute period onset after administration of APAP (Figure 1). Other fractions did not significantly reduce the level of cytolytic enzymes.

For example, the following enzyme levels were detected in the control group at $4 \mathrm{hrs:} \mathrm{GPT} \mathrm{(blue}$ curve) - 3,322.0 $\pm 213.0 \mathrm{U} / \mathrm{L}$, GOT (red curve) 1,285.5 $\pm 98.2 \mathrm{U} / \mathrm{L}$, which exceeded the intact control levels 29.8- and 12.2-fold, respectively. In a group of animals receiving the $10-30 \mathrm{kDa}$ hcMSC fraction, the GPT and GOT levels were 3.4- and 2-fold higher, respectively, as compared to those in the intact control. In animals receiving the $10-30 \mathrm{kDa}$ ncMSC fraction, a significantly greater increase in the enzyme levels was observed compared to hcMSC-treated animals, which exceeded the intact control levels 12.1- (GPT) and 3.1-fold (GOT), respectively (Figure 2).

\section{Mass spectrometry}

A mass spectrometry analysis of the proteins contained in isolated fractions showed a sharp increase in levels of the following proteins in the 10-30 $\mathrm{kDa}$ hcMSC fraction (Table 1) as compared to ncMSC: elongation factor 1-delta (21.5 $\mathrm{kDa})$, platelet-activating factor acetylhydrolase $(22.1 \mathrm{kDa})$, and nucleophosmin $(30 \mathrm{kDa})$. In addition, the concentrations of 10 proteins increased: for tropomyosin $(28.3 \mathrm{kDa})$, it changed 13.1-fold; for thioredoxin (Trx) $(11.7 \mathrm{kDa}), 1.7-$ fold; for myotrophin (12.9 kDa), 3.6-fold; for superoxide dismutase [Cu-Zn] (15.9 kDa), 3.1-fold, for macrophage migration inhibitory factor $(12.5 \mathrm{kDa}), 1.4$-fold; for galectin-1 (14.9 kDa), 1.3-fold; for nucleoside diphosphate kinase (17.2 kDa), 1.8-fold; for peptidyl-prolyl cis-trans isomerase $(18.0 \mathrm{kDa}), 1.5$-fold, for Rho GDP-dissociation inhibitor 1 (23.4 kDa), 1.4-fold and for myosin light polypeptide $6(17.0 \mathrm{kDa}), 1.2$-fold.

\section{Histology}

An analysis of the histological data obtained in control animals revealed signs of acute injury to liver tissue and pronounced centrilobular liver congestion (Figure 3A-B). The blood-congested areas occupied from $1 / 2$ to $2 / 3$ of the lobular areas, sometimes merging with each other. The central parts of the lobules were characterized by discomplexation of the hepatic

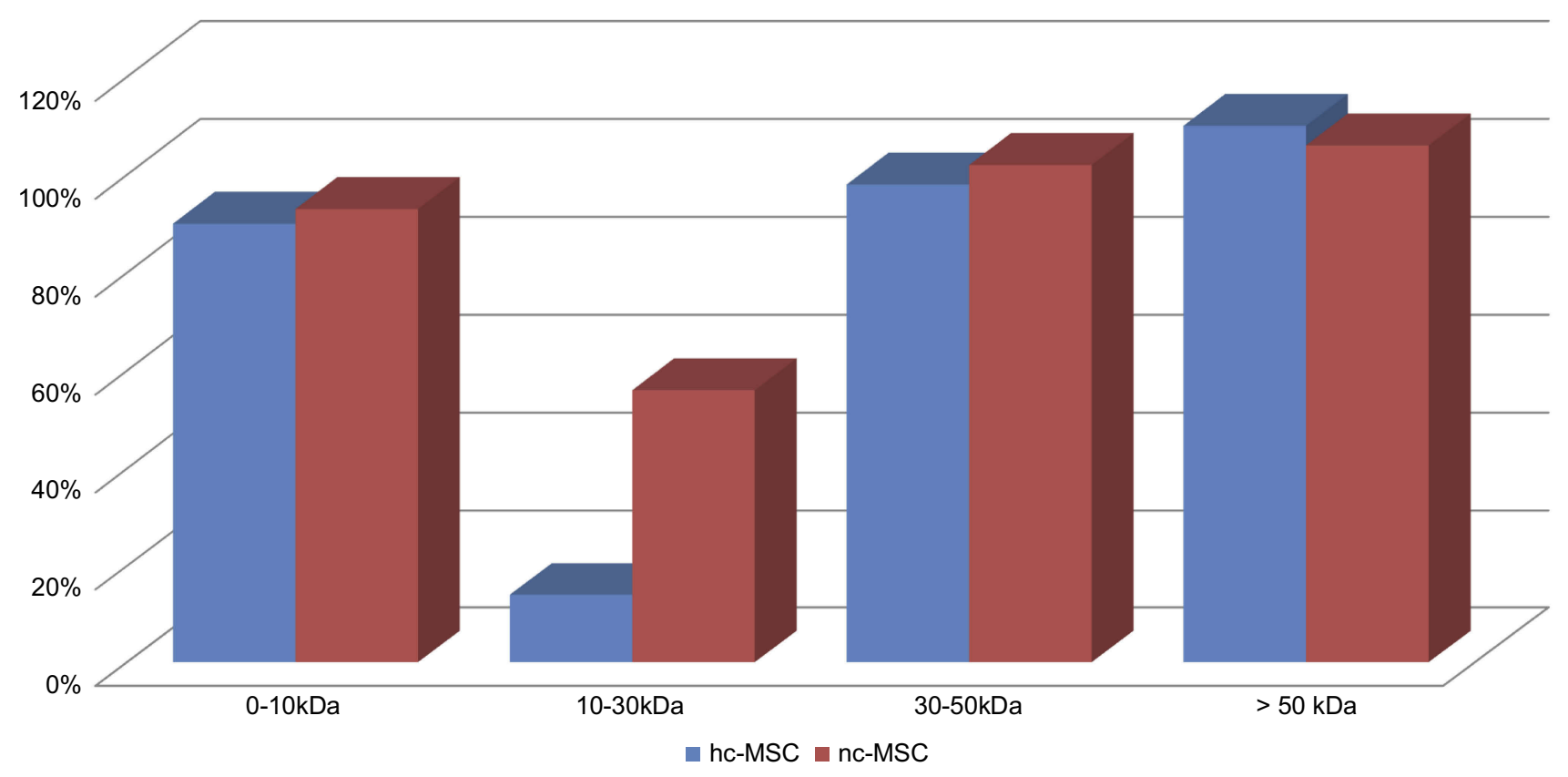

Figure I Activities of liver enzymes GPT in animals treated with hcMSC or ncMSC 4 hrs after administration of acetaminophen.

Abbreviations: hc-MSC, MSCs cultured under hypoxic $\left(10 \% \mathrm{O}_{2}\right.$ hc-MSC) condition; nc-MSC MSCs cultured under normal $\left(21 \% \mathrm{O}_{2}\right.$ nc-MSC) condition. 


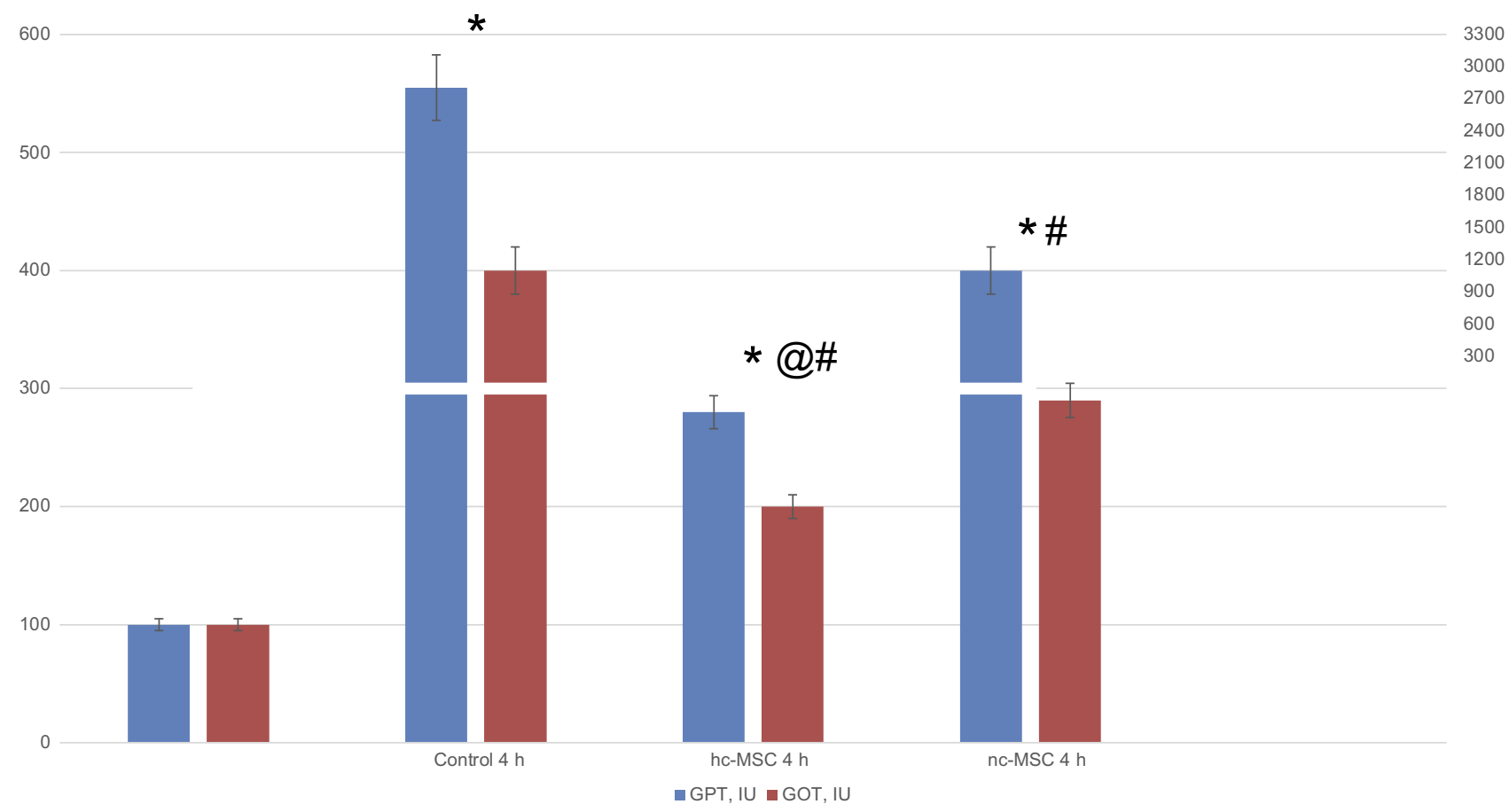

Figure 2 Activities of liver enzymes GPT and GOT in the control group and in animals treated with hcMSC (10-30 kDa) or ncMSC (I0-30 kDa) after the administration of acetaminophen.

Notes: * $p<0.05$ Compared with intact control. @p<0.05 Compared with 24 h. \#p $<0.05$ Compared with Control group.

Abbreviations: hc-MSC, MSCs cultured under hypoxic ( $10 \% \mathrm{O}_{2}$ hc-MSC) condition; nc-MSC MSCs cultured under normal ( $21 \% \mathrm{O}_{2}$ nc-MSC) condition.

cords and destruction of some hepatocytes accompanied by karyorrhexis. Homogenization and total distinct small-droplet fatty degeneration, with a tendency to a fat droplet increase, was observed in the cytoplasm of intact hepatic cells of these lobular parts. Hepatocytes at the periphery of lobules were in the state of granular protein dystrophy and small-droplet obesity. There was a coarse and granular distribution of chromatin in the nuclei of these cells. Single hepatocytes with a large hyperchromic nucleus and binuclear cells were found among them. Dilation and congestion of the hepatic veins were observed in some specimens.

An analysis of the histological data obtained for animals injected with the 10-30 kDa ncMSC fraction compared to those in the control group revealed that the cord structure of hepatic lobules was preserved (Figure 3C-D). Congestion was less pronounced. The sinusoids at the

Table I Results of the analysis of ncMSC and hcMSC (mass spectrometry)

\begin{tabular}{|c|c|c|c|c|}
\hline \multirow[t]{2}{*}{ Protein } & \multirow[t]{2}{*}{ Mol. weight [kDa] } & \multirow{2}{*}{$\begin{array}{l}\text { I0-30 hcMSC } \\
\text { Intensity, } x 10^{3}\end{array}$} & \multirow{2}{*}{$\begin{array}{l}\text { I0-30 ncMSC } \\
\text { Intensity, } x 10^{3}\end{array}$} & \multirow[t]{2}{*}{ hcMSC/ncMSC } \\
\hline & & & & \\
\hline Elongation factor I-delta & 21,478 & 952 & 0 & High \\
\hline Platelet-activating factor acetylhydrolase & 22,056 & 1071 & 0 & High \\
\hline Nucleophosmin & 29,524 & 2282 & 0 & High \\
\hline Tropomyosin & 28,343 & 965 & 74 & 13.1 \\
\hline Thioredoxin & 11,675 & 4,219 & 2,481 & 1.7 \\
\hline Myotrophin & $|2,86|$ & 2,047 & 568 & 3.6 \\
\hline Superoxide dismutase $[\mathrm{Cu}-\mathrm{Zn}]$ & 15,942 & 1,613 & 515 & 3.1 \\
\hline Macrophage migration inhibitory factor & 12,504 & 595 & 425 & 1.4 \\
\hline Galectin-I & 14,866 & 63,682 & 48,986 & 1.3 \\
\hline Nucleoside diphosphate kinase & 17,208 & 3,936 & 2,225 & 1.8 \\
\hline Peptidyl-prolyl cis-trans isomerase & $|7,97|$ & 67,247 & 45,105 & 1.5 \\
\hline Rho GDP-dissociation inhibitor I & 23,407 & 12,053 & 8,576 & 1.4 \\
\hline Myosin light polypeptide 6 & $|6,96|$ & 2,932 & $2,4 \mid 4$ & 1.2 \\
\hline
\end{tabular}




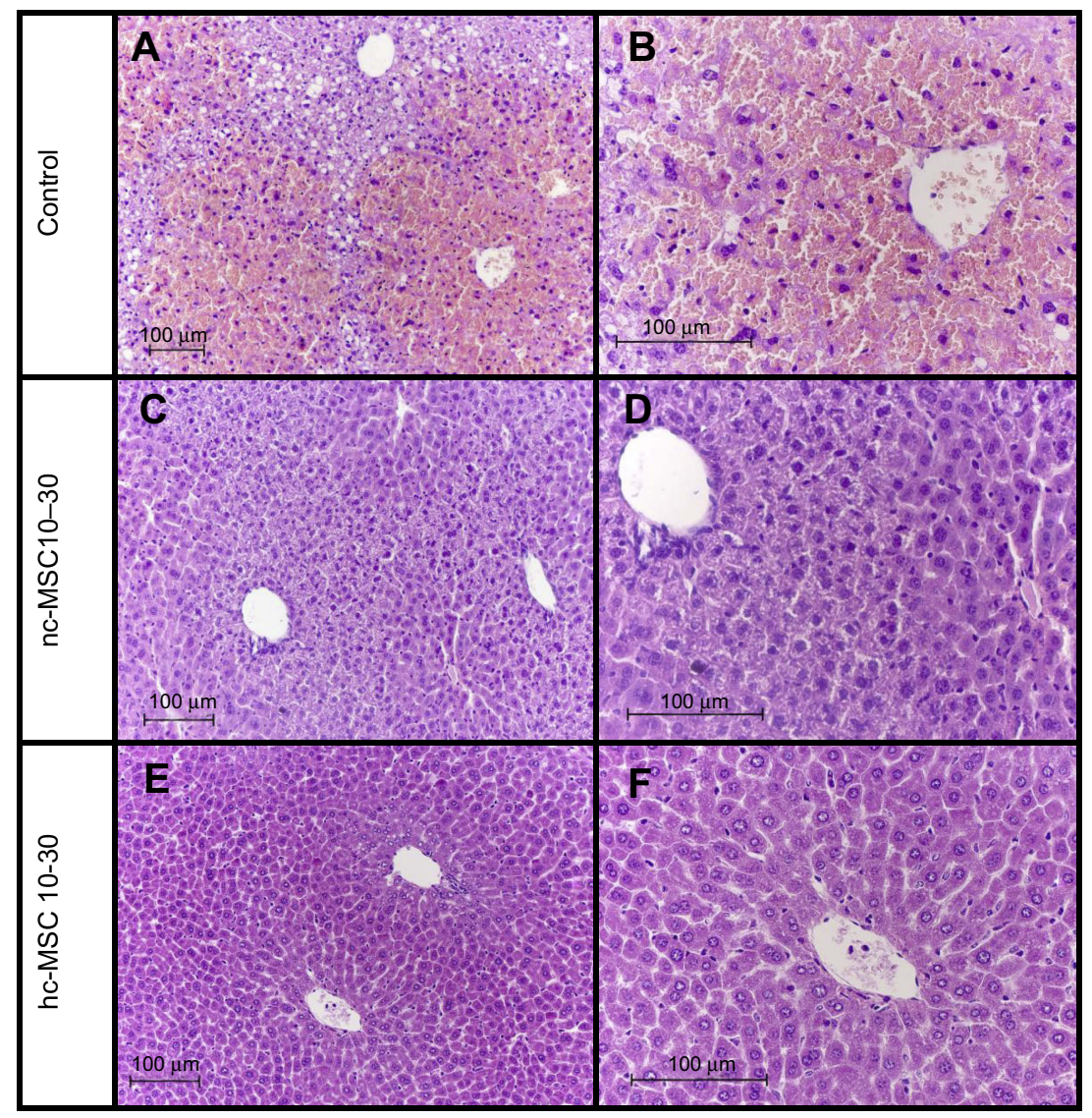

Figure 3 Histology of mouse liver after acetaminophen injection ( $270 \mathrm{mg} / \mathrm{kg})$, stained with hematoxylin and eosin. Control 4 hrs after the injection (A) $\times 160 ;(\mathbf{B}) \times 400 ; 4 \mathrm{hrs}$ after acetaminophen injection $(270 \mathrm{mg} / \mathrm{kg})$ and simultaneous treatment with ncMSC (10-30 kDa) protein compositions (C) x200; (D) $\times 400 ; 4$ hrs after acetaminophen injection $(270 \mathrm{mg} / \mathrm{kg})$ and simultaneous treatment with hcMSC (10-30 kDa) protein compositions (E) $\times 200 ;(\mathbf{F}) \times 400$.

Abbreviations: hc-MSC, MSCs cultured under hypoxic ( $10 \% \mathrm{O}_{2}$ hc-MSC) condition; nc-MSC MSCs cultured under normal $\left(21 \% \mathrm{O}_{2}\right.$ nc-MSC) condition.

periphery of lobules were not enlarged. The cell membranes of hepatocytes in this area retained clear contours. The hepatocyte cytoplasm was characterized by pulverized obesity. A coarse chromatin distribution was detected in the cell nuclei. Single nuclei had a vesicular structure. There were rare binuclear hepatocytes.

The morphological picture of the liver in animals injected with the hcMSC fraction was characterized by the most favorable appearance: congestion of the lobules was usually not observed (Figure 3E-F). The cord structure of lobules was preserved. The cytoplasm of centrilobular hepatocytes presented with signs of swelling as well as granular protein and dusty fatty degeneration. The outer cell membranes had distinct contours. The nuclei of hepatocytes were characterized mainly by a fine chromatin distribution. At the periphery of lobules, there were an increased number of cells with a uniform chromatin distribution and large hyperchromic nuclei. There were a greater number of binuclear cells.
Table 2 The morphometric analysis revealed that there was pronounced injury to liver tissue $4 \mathrm{hrs}$ after acetaminophen introduction

\begin{tabular}{|l|l|l|l|}
\hline Parameter & Control & $\begin{array}{l}\text { ncMSC } \\
\text { I O-30 kDa }\end{array}$ & $\begin{array}{l}\text { hcMSC } \\
\text { I 0-30 kDa }\end{array}$ \\
\hline Score (mean) & 4.45 & 3.925 & 3.075 \\
Standard error & 0.08 & 0.08 & 0.04 \\
Standard deviation & 0.50 & 0.53 & 0.27 \\
Variance & 0.25 & 0.28 & 0.07 \\
Number of & 40 & 40 & 40 \\
measurements & & & \\
\hline
\end{tabular}

Abbreviations: hc-MSC, MSCs cultured under hypoxic ( $10 \% \mathrm{O}_{2}$ hc-MSC) condition; nc-MSC MSCs cultured under normal $\left(21 \% \mathrm{O}_{2}\right.$ nc-MSC) condition.

The morphometric analysis revealed that there was pronounced injury to liver tissue $4 \mathrm{hrs}$ after APAP introduction (Table 2). In this case, introduction of the 10-30 $\mathrm{kDa}$ hcMSC fraction exerted the maximum protective 
effect, significantly differing from the control group and the 10-30 kDa ncMSC-treated group.

\section{Discussion}

Treatment of APAP-induced ALF requires serious medical measures and, in some cases, liver transplantation. The use of NAC as an antidote is associated with a number of side effects. ${ }^{4}$ In addition, the therapeutic window for NAC administration is rather narrow, which, as has been shown, reduces its efficacy in APAP-treated mice. ${ }^{5}$ A number of studies have demonstrated that MSCs can be an effective method for the prevention and treatment of APAP-induced ALF. However, cell transplantation has several limitations. The use of autologous MSCs in the acute period is actually impossible because a sufficient dose of cells should be introduced within the first hours after an event to achieve a positive clinical effect. In this case, it is necessary to emphasize the apparent dose dependence of the clinical effect on the amount of transplanted cellular material. ${ }^{14}$

The use of a high dose of allogeneic cells may lead to severe complications and immune dysfunction. ${ }^{15,16}$

One of the possible ways to solve this problem may be the use of paracrine factors produced by stem cells. The effectiveness of their use was demonstrated in our previous work. ${ }^{9}$ However, we believe that the use of the total fraction is not reasonable.

Our findings demonstrated that the $10-30 \mathrm{kDa}$ fraction had a protective effect via inhibition of APAPinduced ALF. At the same time, the medium fraction obtained under low oxygen content conditions was maximally effective.

One of the possible causes for this effect may be a quantitative difference in the peptide composition, which was detected by LC-MS. As can be seen from Table 1, concentrations of 10 proteins increased from 1.2- to 13.1fold. The concentration of three proteins increased to a detectable level.

According to the literature data, three of the presented proteins (galectin-1, Trx, SOD) can reduce the degree of injury during the development of APAP-induced ALF.

Galectin-1 is a protein secreted by stem cells into culture medium; it inhibits the inflammatory response by suppressing proliferation of $\mathrm{T}$ lymphocytes. ${ }^{17,18} \mathrm{~A}$ study by Ryu et al demonstrated that tonsil-derived MSCs were able to inhibit the development of ConA-induced hepatitis via the production of galectin-1. The authors attributed this effect to blockade of CD4+ T lymphocyte activation by galectin-1. ${ }^{14}$

Trx is a protein that regulates the production of reactive oxygen species and is produced in response to oxidative stress. ${ }^{19,20}$ This protein exerts an anti-inflammatory effect via inhibition of neutrophil chemotaxis. ${ }^{21}$ Okuyama et al reported that an increase in Trx expression prevented the development of acute hepatitis caused by thioacetamide or LPS. ${ }^{22}$ Taneka et al demonstrated that albumin-conjugated Trx effectively reduced the degree of liver injury caused by APAP. $^{23}$

It should be noted that a decrease in the oxygen level during MSC cultivation leads to a sharp (2.6-fold) increase in MIF production. According to the literature, this proinflammatory factor increases the degree of injury to liver tissue. ${ }^{24}$ However, on the other hand, MIF was shown to increase survival of MSCs in hypoxic conditions protecting cells from hypoxia-induced apoptosis and reducing the level of oxidative stress ${ }^{25}$ Perhaps, this mechanism may occur in hepatocytes exposed to APAP.

\section{Ethical considerations}

Ethical clearance for the study was obtained from the Institute of Cell Biophysics of the Russian Academy of Sciences prior to study initiation. The study was conducted in compliance with the principles of the Declaration of Helsinki. In addition to the approval from the Institute of Cell Biophysics of the Russian Academy of Sciences, the maintenance and ethical treatment of laboratory animals was done in accordance with Guidelines of the European Convention for the Protection of Vertebrate animals used for experiments and Guidelines for working with laboratory animals in ICB RAS, Order N39 from December 30, 2018.

\section{Acknowledgments}

This study was supported by the Russian Foundation for Basic Research (grant no. 17-07-00439). The mass spectrometry experiments, data analysis, and specific protein identification were supported by the Russian Science Foundation grant no. 16-15-10431.

\section{Disclosure}

Stanislav Pekov report grants from the Russian Scientific Foundation, during the conduct of the study. The authors report no other conflicts of interest in this work. 


\section{References}

1. Lee WM. Drug-induced acute liver failure. Clin Liver Dis. 2013;17:575-586. doi:10.1016/j.cld.2013.07.001

2. Zimmerman HJ, Maddrey WC. Acetaminophen (paracetamol) hepatotoxicity with regular intake of alcohol: analysis of instances of therapeutic misadventure. Hepatology. 1995;22:767-773. doi:10.1002/(ISSN)1527-3350

3. Wootton FT, Lee WM. Acetaminophen hepatotoxicity in the alcoholic. South Med J. 1990;83:1047-1049. doi:10.1097/00007611199009000-00017

4. Sandilands EA, Bateman DN. Adverse reactions associated with acetylcysteine. Clin Toxicol. 2009;47:81-88. doi:10.1080/ 15563650802665587

5. Saito C, Zwingmann C, Jaeschke H. Novel mechanisms of protection against acetaminophen hepatotoxicity in mice by glutathione and $\mathrm{N}$ acetylcysteine. Hepatology. 2010;51:246-254. doi:10.1002/ hep. 23267

6. Salomone F, Barbagallo I, Puzzo L, Piazza C, Li Volti G. Efficacy of adipose tissue-mesenchymal stem cell transplantation in rats with acetaminophen liver injury. Stem Cell Res. 2013;11(3):1037-1044. doi:10.1016/j.scr.2013.07.003

7. Stock P, Brückner S, Winkler S, Dollinger MM, Christ B. Human bone marrow mesenchymal stem cell-derived hepatocytes improve the mouse liver after acute acetaminophen intoxication by preventing progress of injury. Int J Mol Sci. 2014;15:7004-7028. doi:10.3390/ ijms15047004

8. Khubutiya MS, Vagabov AV, Temnov AA, Sklifas AN. Paracrine mechanisms of proliferative, anti-apoptotic and anti-inflammatory effects of mesenchymal stromal cells in models of acute organ injury. Cytotherapy. 2014;16(5):579-585. doi:10.1016/j.jcyt.2013.07.017

9. Temnov AA, Vagabov AV, Sklifas AN, et al. The role of mesenchymal stromal cells in the repair of acute organ injury. In: Atkinson K, editor. The Biology and Therapeutic Application of Mesenchymal Cells Blackwell. Hoboken: Blackwell (John Wiley \& Sons); 2016:496-523.

10. Lee JW, Fang X, Gupta N, Serikov V, Matthay MA. Allogeneic human mesenchymal stem cells for treatment of E. coli endotoxininduced acute lung injury in the ex vivo perfused human lung. Pnas. 2009;106(38):16357-16362. doi:10.1073/pnas.0907996106

11. MSh K, Temnov AA, Vagabov AV, et al. Effect of conditioned medium and bone marrow stem cell lysate on the course of acetaminophen-induced liver failure. Bull Exp Biol Med. 2015;159(1):118123. doi:10.1007/s10517-015-2905-x

12. Yagi H, Soto-Gutierrez A, Navarro-Alvarez N, et al. Reactive bone marrow stromal cells attenuate systemic inflammation via sTNFR1. Mol Ther. 2010;18(10):1857-1864. doi:10.1038/mt.2010.155
13. Kosyreva AM, Simonova EY. Histophysiology of the immune system of Wistar rats at delayed terms after ovariectomy. Bull Exp Biol Med. 2013;155(5):697-700.

14. Ryu KH, Kim SY, Kim YR, et al. Tonsil-derived mesenchymal stem cells alleviate concanavalin A-induced acute liver injury. Exp Cell Res. 2014;326(1):143-154. doi:10.1016/j.yexcr.2014.06.007

15. Sundin M, Orvell C, Rasmusson I, Sundberg B, Ringdén O, Le Blanc $\mathrm{K}$. Mesenchymal stem cells are susceptible to human herpes viruses, but viral DNA cannot be detected in the healthy seropositive individual. Bone Marrow Transplant. 2006;37(11):1051-1059. doi:10.1038/sj.bmt.1705368

16. Amariglio N, Hirshberg A, Scheithauer BW, et al. Donor-derived brain tumor following neural stem cell transplantation in an ataxia telangiectasia patient. PLoSMed. 2009;6(2):e1000029.

17. Gieseke F, Böhringer J, Bussolari R, Dominici M, Handgretinger R, Müller I. Human multipotent mesenchymal stromal cells use galectin-1 to inhibit immune effectors cells. Blood. 2010;116(19):37703779. doi:10.1182/blood-2010-02-270777

18. Lepelletier Y, Lecour BA, Renand A, et al. Galectin-1 and semaphorin-3A are two soluble factors conferring T-cell immunosuppression to bone marrow mesenchymal stem cell. Stem Cells Dev. 2010;9 (7):1075-1079. doi:10.1089/scd.2009.0212

19. Holmgren A. Thioredoxin and glutaredoxin systems. J.Biol.Chem. 1989;264:13963-13966.

20. Nakamura T, Nakamura H, Hoshino T, Ueda S, Wada H, Yodoi J. Redox regulation of lung inflammation by thioredoxin. Antioxid Redox Signaling. 2005;7:60-71. doi:10.1089/ars.2005.7.60

21. Nakamura H, Herzenberg LA, Bai J, et al. Circulating thioredoxin suppresses lipopolysaccharide-induced neutrophil chemotaxis. Proc Natl Acad Sci U S A. 2001;98:15143-15148. doi:10.1073/pnas.191498798

22. Okuyama H, Nakamura H, Shimahara $Y$, et al. Overexpression of thioredoxin prevents acute hepatitis caused by thioacetamide or lipopolysaccharide in mice. Hepatology. 2003;37:1015-1025. doi:10.1053/jhep.2003.50203

23. Tanaka R, Ishima Y, Maeda H, et al. Albumin fusion prolongs the antioxidant and anti-inflammatory activities of thioredoxin in mice with acetaminophen-induced hepatitis. Mol Pharm. 2014;11(4):12281238. doi: $10.1021 / \mathrm{mp} 400690 \mathrm{v}$

24. Bourdi M, Reilly TP, Elkahloun AG, George JW, Pohl LR. Macrophage migration inhibitory factor in drug-induced liver injury: a role in susceptibility and stress responsiveness. Biochem Biophys Res Commun. 2002;294(2):225-230. doi:10.1016/S0006-291X(02) 00466-7

25. Xia W, Xie C, Jiang M, Hou M. Improved survival of mesenchymal stem cells by macrophage migration inhibitory factor. Mol Cell Biochem. 2015;404(1-2):11-24. doi:10.1007/s11010-015-2361-y
Hepatic Medicine: Evidence and Research

\section{Publish your work in this journal}

Hepatic Medicine: Evidence and Research is an international, peerreviewed, open access journal covering all aspects of adult and pediatric hepatology in the clinic and laboratory including the following topics: Pathology, pathophysiology of hepatic disease; Investigation and treatment of hepatic disease; Pharmacology of drugs used for the treatment of hepatic disease. Issues of patient safety and quality of care will also be considered. The manuscript management system is completely online and includes a very quick and fair peer-review system, which is all easy to use. Visit http://www.dovepress.com/ testimonials.php to read real quotes from published authors. 